REVISTA ANDALUZA DE ANTROPOLOGÍA.

NÚMERO 7: GITANOS/ROMA:AUTO-PRODUCCIÓNCULTURAL Y CONSTRUCCIÓN HISTÓRICO-POLÍTICA

SEPTIEMBRE DE 2014

ISSN 2174-6796

[pp. 7-22]

http://dx.doi.org/10.12795/RAA.2014.i07.01

Fecha de Recepción: 25-03-2014

Fecha de Aceptación: 25-04-2014

\title{
CONSTRUCCIÓN Y USO SOCIAL DE LA REPRESENTACIÓN DE LOS GITANOS POR EL PODER FRANQUISTA 1936-1975 ${ }^{1}$
}

\author{
Xavier Rothea \\ Université Paul-Valéry Montpellier III
}

\section{Resumen.}

La historia de los Gitanos españoles parece detenerse en 1783, cuando Carlos III otorgó, teóricamente, los mismos derechos a los Gitanos que al resto de los españoles. La palabra Gitanos desaparece de los textos oficiales y los Gitanos parecen sobrevivir únicamente en la literatura romántica de los viajeros franceses. Aunque no disponemos de muchos archivos, existe otro tipo de documentos en la literatura o los medios de comunicación que nos permite tener una mejor idea de la posición que ocupa esta comunidad en la sociedad española, centrándome en la reciente época franquista. Mi tentativa consiste en abordar a través de la investigación histórica el problema de la construcción y el uso social y político de la representación de los Gitanos en el seno de un Estado franquista católico-reaccionario y nacionalista extremo.

Palabras clave.

Gitanos, franquismo, historia, representación.

\section{Abstract.}

The history of Spanish Gypsies seems to stop in 1783, when Carlos III granted theoretically 
equal rights to Gypsies than the rest of the Spaniards. The word Gypsies disappear from official texts and the Gypsies seem to survive only in the romantic literature of the French travelers. Although we do not have many files there are other documents in the literature or media that allow us to get a better idea of the position of this community in Spanish society, focusing on the recent Franco era. My attempt is to address through historical research the problem of the construction and use of social and political representation of Roma within a Catholic-reactionary and radical nationalistic franquista State.

\section{Keywords.}

Gypsies, franquism, history, representation.

La historia de los Gitanos españoles parece detenerse en 1783, cuando Carlos III otorgó, teóricamente, los mismos derechos a los Gitanos que al resto de los españoles. La palabra Gitanos desaparece de los textos oficiales y los Gitanos parecen sobrevivir únicamente en la literatura romántica de los viajeros franceses. Sabemos poco acerca de los Gitanos durante los siglos XIX y XX en sus respectivas fases de grandes convulsiones en la sociedad española. No obstante, los Gitanos constituían la minoría más importante en el país. Y en ausencia de cualquier mención legal, excepto en un artículo en el reglamento de la Guardia Civil $^{2}$ que data de 1852, y careciendo de documentos escritos elaborados por los propios Gitanos, esta historia no es fácil de escribir. Aunque no disponemos de muchos archivos, existe otro tipo de documentos en la literatura o los medios de comunicación que nos permite tener una mejor idea de la posición que ocupa esta comunidad en la sociedad española. Este es un primer paso para un mejor conocimiento de la historia más reciente. En este texto, mi tentativa consistirá en abordar el problema de la construcción y el uso social y político de la representación de los Gitanos en el seno de un Estado franquista católico-reaccionario y nacionalista extremo, y desarrollaré las conclusiones de mi trabajo específico. Para ello, previamente, señalaré algunos cuestionamientos sobre el método utilizado para la investigación histórica.

\section{HIPÓTESIS, ÁREAS DE INVESTIGACIÓN, MÉTODOS DE TRABAJO}

De inicio, es imprescindible aportar datos sobre el método empleado en mi investigación. Con el fin de contextualizar este estudio cabe realizar un balance sobre el conocimiento general y aceptado en relación con el régimen franquista en España y en relación a los Gitanos españoles, para posteriormente confrontar uno con otro. ¿Qué es lo que sabemos sobre el régimen franquista y en qué medida puede tener relación con los Gitanos españoles?

2. Policía militar española. 
Entre 1936 y 1975, España estaba bajo el régimen franquista con un régimen de extrema derecha y nacionalista. En los años 30 y 40, todos los regímenes fascistas eran al mismo tiempo regímenes racistas. España no participó en el genocidio y el asesinato en masa de Judíos y Gitanos en Europa (Benassar, 1992), pero el antisemitismo estaba incorporado en el aparato del Estado y en muchos grupos de la población (Álvarez Chillida, 2002; Rozenberg, 2006). La especificidad del régimen franquista en el grupo de los regímenes fascistas -o casi fascistas- es su carácter católico fundamentalista. Este carácter se expresa en el concepto de "hispanidad" nacido a finales del siglo XIX después de la pérdida de Cuba, la última posesión del imperio colonial español. La noción de "hispanidad" empleada por los franquistas representaba la exaltación de la "raza" española definida teóricamente por su carácter católico. El régimen franquista, establecido sobre las ruinas de una democracia que aspiraba a construir nuevas relaciones sociales, debía proponer otros modelos y ejemplos sociales y políticos que pudieran ilustrar su concepción del "pueblo español". Los Gitanos eran la única minoría visible considerada como un grupo racial diferente. Su número estimado era entre 300.000 y 400.000 personas. Vivían en su mayoría en el sur de España -la mayor parte en Andalucía-, en Cataluña y Madrid, pero se encontraban comunidades en toda España. Muy pocos de ellos, alrededor del 5\%, eran nómadas. Durante los años 50 y los años 60, debido a las dificultades económicas y la modernización de la agricultura, un número creciente de Gitanos abandonaron el campo en dirección a las grandes ciudades. Muchos de ellos tuvieron que vivir en los barrios pobres de Madrid, Barcelona o Valencia. La mayoría de los Gitanos vivían en la miseria y la mayoría de ellos eran parte de la clase social más pobre de la sociedad, que habitaba en "chabolas" o cuevas, sobre todo en Andalucía, sin agua ni electricidad. En 1968, y según un informe de la asociación católica Caritas, el 85\% de los Gitanos eran analfabetos y el 90\% de la "gitanillos" no habían cursado la escuela primaria. Respecto a esta situación de pobreza, desde el siglo XVI los Gitanos tenían fama de ladrones y herejes. Sin embargo, algunos Gitanos se vinculaban con el flamenco o el comercio, representando una pequeña minoría rica y socialmente reconocida. En relación a ello, los Gitanos fueron una de las manifestaciones del "folklore español" en la promoción turística implementada en este período. Las Cuevas de Granada, el barrio de Triana o el "tablao flamenco" en Madrid fueron los lugares imprescindibles para el visitante turístico en España.

Poner en relación estos hallazgos suscita muchas preguntas que pueden definir las áreas de investigación. En primer lugar, ¿ esta ausencia de participación en el asesinato en masa y el genocidio de los Judíos y los Roma europeos significaría un rechazo institucional de la discriminación hacia las minorías y en especial hacia la única "minoría visible", los Gitanos? Los estudios recientes de Rozenberg muestran que el régimen franquista no siempre tuvo una actitud de protección respecto a los Judíos tal como los partidarios 
de Franco esgrimieron a lo largo de los años (Rozenberg, 2006: 135-166). En segundo lugar, un nuevo régimen debía construir nuevas normas sociales también. El franquismo no podía gobernar sólo con la represión y el control social. También era necesario el apoyo de gran parte de la población, sobre todo después de una guerra civil, con lo cual el régimen propuso normas sociales muy unificadoras. ¿Las representaciones aplicadas a los Gitanos eran compatibles con las normas sociales franquistas? En tercer lugar, ¿̇la alianza entre el Estado y la Iglesia católica durante este período y la proclamación por el poder de una concepción no biológica de la "raza hispana" en torno a la noción de la Hispanidad constituían un antídoto suficiente contra el racismo clásico? En cuarto lugar, ¿la representación folclórica de los Gitanos proyectada hacia el mundo era el único uso de la imagen de los Gitanos por parte del Estado franquista?

Este cuestionamiento posibilita la construcción de hipótesis basadas en convicciones personales y pistas concordantes que debemos confirmar por medio del estudio y análisis de los documentos disponibles. Podemos suponer que: 1) el régimen franquista, como régimen nacionalista, no pudo prescindir de una representación racista de los Gitanos como unos "otros internos"; 2) Al igual que otros países de Europa, antes y durante la Segunda Guerra Mundial, España utilizó la justificación biológica para la criminalización de los Gitanos; 3) Los Gitanos fueron esgrimidos como un contra-ejemplo social y la representación folclórica se construyó únicamente para su uso promocional respecto a los turistas; 4) Este contra-ejemplo contribuía a aglutinar una sociedad desgarrada por una guerra civil: los Gitanos como un nuevo enemigo social para olvidar al enemigo político.

Estas hipótesis convergen en una temática común: la construcción y el uso de la representación de los Gitanos en la sociedad franquista. El trabajo sobre los Archivos ofrece la oportunidad de iluminar el proceso de la construcción ideológica y el uso social / político de esta representación. Para confirmar o desestimar esta hipótesis es necesario consultar las investigaciones en torno a tres temas. En primer lugar, la representación de los Gitanos en la legislación y el discurso del régimen franquista, así como también en la antropología "institucional"; en segundo lugar, la difusión de la representación "oficial" en los medios de comunicación bajo control estatal; y en tercer lugar, el acuerdo, la discrepancia y la evolución entre el régimen y la Iglesia católica en relación a la representación de los Gitanos.

\section{ARCHIVOS Y BIBLIOGRAFÍA}

Disponemos de una amplia bibliografía para aproximarnos a la historia de los Gitanos antes del siglo XIX (Leblon, 1985; Sánchez Ortega, 1988, 1991; Gómez Alfaro, 1994). Los historiadores revelan la discriminación histórica contra los Gitanos desde la Pragmática de Medina del Campo (1499) hasta la legislación asimilacionista de Carlos III en 1783. 
Leblon saca a la luz el caso especial de los Gitanos de Andalucía y lo particular de la interculturalidad en esta zona desde el siglo XVII, cuando los Gitanos reemplazaron a los moriscos en el trabajo en el campo. Según Leblon, el flamenco es el resultado de esta interculturalidad. Recientemente, durante los 30 últimos años, se han editado numerosas publicaciones acerca de la situación de los Gitanos en España tras la dictadura (Gay y Blasco, 1999; Teresa San Román, 1976... y otros). Los estudios sociológicos y etnológicos sobre zonas de marginalidad, la discriminación o las políticas públicas se han multiplicado, a tal punto que no podemos mencionarlos a todos en la bibliografía.

Por el contrario, son muy pocas las publicaciones y la información disponible sobre la situación en el siglo XIX, la Segunda República, la Guerra Civil y el régimen franquista. En cuanto al siglo XIX, no podemos considerar a Borrow y las narrativas de los románticos franceses como estudios científicos sobre la situación real de los Gitanos en el país. Antes de 2009, algunos estudios fueron publicados en relación a la situación de los Gitanos bajo la dictadura franquista. Un artículo de David Martín en la revista gitana "I Tchachipen" y los estudios de Nancy Thede, llegan a la misma conclusión en relación a la no participación de la comunidad gitana en la guerra civil (Martín, 2005; Thede, 1999). Sólo un libro existe en relación directa con la comunidad durante el período franquista: el estudio de Marie Franco del periódico sensacionalista «El Caso» (1952-1962), que incluye una parte sobre la representación de los Gitanos en esta publicación (Franco, 2006). En 2009 se publicó el primer libro sobre los Gitanos durante la guerra civil. Esta publicación fue el resultado del trabajo de la asociación de mujeres gitanas de Granada "Romi" (Fernández Fernández, Flor Heredia, Rodríguez Padilla, 2009) y aporta nueva información sobre la situación de los Gitanos en la guerra civil y en esta área.

Como vemos, la bibliografía no puede responder por completo a la problemática planteada pero ofrece un marco en el que el investigador puede calibrar la importancia de las fuentes de información. Una vez realizado este paso, debemos seleccionar las fuentes que pueden proporcionar respuestas. Aquí, tres tipos diferentes de documentos resultan esenciales:

Para empezar, tenemos que examinar, en primer lugar, las fuentes oficiales del poder, los ministerios o la administración. Informes oficiales, leyes y normas, así como comentarios jurídicos tienen que ser considerados. Otras fuentes que a menudo son olvidadas: los boletines profesionales de las instituciones franquistas. La revista de la Guardia Civil (1944-1978), Policía (1942-1962), Policía Nacional (1962-1984) del cuerpo de policía o la Revista de la Escuela de Estudios Penitenciarios (1946-1961) de la Administración de Justicia. También podemos añadir a las fuentes oficiales, el libro publicado en la colección “Temas españoles", dirigida por el Ministerio de Información. Después hay que estudiar las fuentes no gubernamentales en general, y en particular, los estudios 
escolares y libros de divulgación, la prensa no especializada y especializada, así como la literatura infantil publicados entre 1936 y 1975. En relación a la prensa, los periódicos nacionales más importantes como $A B C$, Blanco y negro, Arriba, deben ser consultados por la influencia que tuvieron en la sociedad como El Caso por su gran número de lectores. Los periódicos regionales pueden ser interesantes en los lugares con importantes comunidades gitanas, como es el caso de Ideal de Granada. Por último, tenemos que investigar las fuentes eclesiásticas. Las publicaciones de la Iglesia no fueron sometidas a la censura gubernamental. La institución católica tenía a su disposición muchos periódicos (Ecclesia fue la más leída) y revistas (Cáritas, mundo christiano, Documentación social). Dos revistas en relación a las comunidades gitanas fueron publicados por la iglesia, Pomezia desde 1967 y Diálogo gitano desde 1969. En el mismo tipo de fuentes podemos incluir a los autores eclesiásticos y los informes de asociaciones católicas.

Los hallazgos realizados tras el análisis de estos documentos permiten avanzar algunas conclusiones que pueden responder a una parte de las preguntas iniciales. Estas conclusiones se organizan en torno a cinco ideas principales.

\section{a) La esencialización racista de la "criminalidad gitana" por las autoridades franquistas}

En relación a la lectura de los boletines profesionales de las instituciones franquistas, emerge con claridad una diseminación y apología de la antropología física francesa y alemana en la antropología institucional española desde el inicio del siglo XX hasta 1960. El antropólogo Luis Hoyos de Sainz (1882-1950) fue uno de los fundadores de la antropología española. Sobre la base de las medidas físicas, dividió a los españoles en varios grupos raciales y presentó a los Gitanos como un grupo racial externo (Hoyos de Sainz, 1951). Esta presentación de los Gitanos era comúnmente aceptada por su sucesor y propagada por los informes institucionales. Al mismo tiempo, advertimos la reutilización de la criminología positivista de Lombroso en la justificación de la criminalización de los Gitanos. En su famoso ensayo Criminal Man (1895), el criminólogo italiano escribe sobre los Gitanos como una raza de "ladrones natos" entre los cuales la criminalidad es heredada. En España la criminología positivista fue desarrollada por Rafael Salillas quien considera a los Gitanos como una raza de criminales, no sólo por la sangre sino en relación con su estilo de vida y el nomadismo. Su influencia en los criminólogos franquistas fue muy importante. En sus artículos, los Gitanos son concebidos como criaturas hereditariamente y racialmente criminogénicas. Otras influencias se pueden encontrar en su escritura. A principios de los años 40, la Kriminal biologie y el higienismo racial nazi aparecieron en la criminología franquista (Rothea, 2007). Mezger y Exner, los teóricos de la vertiente jurídica de la criminología biológica nazi y el tristemente famoso Ritter se convirtieron en las autoridades en este ámbito para criminólogos franquistas 
como Valentín Guerra (ver Guerra, 1944) o el doctor Echalecu y Canino3. Algunos de ellos participaron, notablemente Echalecu y Canino, en 1943 en un viaje a Alemania organizado por la Comisión Internacional de la Policía Criminal ${ }^{4}$,en el estudio de los métodos alemanes de la criminología. Durante este viaje, hicieron una visita al Instituto de Biología Criminal con el propio Ritter. Echalecu y Canino (1945) rindió en el REEP un informe entusiasta sobre el tratamiento de los "asociales". La criminología positivista, la criminología biológica, la criminología clásica española y también la antigua tradición literaria de la representación de los Gitanos como "ladrones" naturales, todas convergen hacia la imagen de un gitano "naturalmente" criminal como consecuencia de su "raza".

\section{b) El uso social de la representación criminal de los Gitanos}

Nunca se ha demostrado que los Gitanos sean más criminales que el resto de la población. Ninguna investigación seria y rigurosa se llevó a cabo durante este período en relación a la supuesta criminalidad gitana pero ésta se afirma en todos los tratados y artículos de criminología. ¿Por qué?

Comparada con otros tipos de criminalización en el resto de los países de Europa Occidental durante el mismo período, la actitud franquista es más dura y se apoya en consideraciones racistas. En Francia o Inglaterra, por ejemplo, la hostilidad contra los Gitanos estaba presente tanto en la población como en la policía, pero después de la Segunda Guerra Mundial, nunca ha sido parte de una afirmación institucional, sea cual fuere la realidad del tratamiento. La hostilidad parece más asumida y confirmada por el régimen franquista que por los regímenes democráticos.

Podemos pensar que la "criminalidad de los Gitanos" fue utilizada por el poder en España con un objetivo social y político. Debemos tener en cuenta la situación histórica particular de España en los años 40 y 50. El país estaba dividido por la guerra civil y los antagonismos políticos. Después de una terrible represión que acabó con varios cientos de miles de víctimas, el nuevo régimen necesitaba reunificar al pueblo español en torno a principios comunes. La hostilidad contra los Gitanos, presentados como los más grandes criminales del país, es una de las formas empleadas para construir nuevos enemigos sociales y "raciales" en lugar de los antiguos enemigos políticos. Los franquistas contaban con la hostilidad tradicional desarrollada hacia la monarquía y la Iglesia desde el siglo XVI, pero también con los "nuevos" postulados racistas. Como los criminales, los Gitanos fueron ubicados al exterior de la sociedad ofreciendo un modelo perfecto de enemigos antisociales. La biologización de la delincuencia era también una manera de ocultar los problemas sociales, la pobreza y la incapacidad del Estado para resolverlos. Los

3. Quien era profesor de psicología criminal en la Academia de Policía de Madrid.

4. Esta Comisión, la cual estaba ubicada en Berlín desde 1938 y liderada por Heydrich, fue el precedente de la Interpol. 
Gitanos eran el perfecto contra-ejemplo para los españoles a la hora de integrar nuevos estándares sociales (véase el gráfico 1). Esta es también una buena manera de asustar a la población para generar la necesidad de seguridad y justificar el estado policial y un fuerte control social. La representación de los Gitanos no fue la única manera de alcanzar estos objetivos, pero constituyó uno de los elementos de la política general.

\section{c) Institucionalización y criminalización de la diferencia}

La criminalización de los Gitanos no fue una construcción sencilla de los franquistas. Fue institucionalizada sobre la base de una "diferencia" esencialista. Esta "diferencia" fue afirmada por una supuesta especificidad de la delincuencia gitana (robo de caballos, mendicidad, hurto en tiendas...). El comercio de caballos era una actividad tradicional de los Gitanos en el campo. Al igual que el resto de sus actividades, ésta parecía especialmente sospechosa para la policía, además, los caballos representaban, en el contexto de la pobreza en España en los 40, una herramienta de trabajo valiosa (Comandante Casillas, 1965). En este contexto también, si bien algunos Gitanos practicaban la mendicidad para sobrevivir, ellos no eran los únicos, pero las autoridades no podían permitir que la gente creyera que los españoles se veían obligados a ejercerla en un estado nacionalcatólico. En muchos textos, el nomadismo se presenta como una especificidad de la comunidad gitana española, pero en realidad sólo el 5\% de ellos practicaban este estilo de vida (Dirección Nacional de Apostolado Gitano, 1968). La legislación real contra el nomadismo que data de 1499 fue muy eficiente (Leblon, 1985). Sin embargo, la policía consideraba esta actividad como práctica general, ya que coincidía con la idea de que los Gitanos eran nómadas con el fin de cometer delitos y escapar de la policía. El Kalo, el lenguaje de los Gitanos españoles, era presentado como el lenguaje del delincuente utilizado para engañar a la policía y al "payo" (Serrano García, 1945:4). Su estilo de vida, sus actividades, su lengua, todo ello simbolizó a los Gitanos como una población peligrosa. Por lo tanto, las autoridades tuvieron que reaccionar frente a la "amenaza" que habían creado ellos mismos frente a la sociedad o para ofrecer a la población la sensación de que podían protegerla.

Los franquistas podían usar tres herramientas legales, y tal vez más, para luchar contra esta “amenaza social”. La primera fue producto de la Segunda República, que legisló en 1934 sobre la peligrosidad social en la normativa llamada "Ley de vagos y maleantes». Esta ley fue hecha por los juristas de izquierda positivistas que pretendían generar más prevención que represión, pero con el etiquetaje de los grupos peligrosos (transeúntes, vagabundos, alcohólicos, prostitutas, homosexuales,...), este texto se convirtió en un arma contra toda persona considerada como un desviado social o político durante la dictadura. Fue una de las pocas leyes de la República conservada por el franquismo. Muchos de los documentos de las autoridades demuestran que los Gitanos fueron incluidos en la categoría de "Vagos" 
(Comandante Casillas, 1965), pero no sabemos en qué proporción esta legislación, que suponía el encarcelamiento preventivo de los que eran considerados como peligrosos, se aplicó o no. En 1971, este texto fue sustituido por la "Ley de peligrosidad y rehabilitación social", que mantiene a los Vagos como grupos peligrosos y a los Gitanos como "Vagos" (Sabater Tomás, 1962; 1972). La tercera herramienta para luchar contra la "criminalidad gitana” fue la Guardia Civil, la Policía Militar Española. Desde 1852, una de las misiones de la Guardia Civil era el seguimiento de los Gitanos y de sus actividades. En un artículo de su reglamento interno, se especifica que los policías deben mantener una estrecha vigilancia sobre los Gitanos y sus movimientos. Este reglamento se reestructuró en 1943 y el artículo sobre los Gitanos se mantuvo. Además, esta obligación era recordada con frecuencia a la guardia en su boletín profesional (Jiménez Rubio, 1948).

El uso social de los Gitanos tuvo una utilidad interna para las autoridades. No era la misma imagen que se ofrecía en las guías turísticas para un uso externo. El régimen no quería asustar a los turistas europeos, por el contrario, los franquistas, que necesitaban el dinero del turismo y querían desarrollar este sector económico, edificaron otra imagen, en la misma línea que los románticos franceses e ingleses capaces de activar la imaginación de los visitantes que esperaban ver Carmen en Sevilla o Granada. No se trataba en este caso de una peligrosa raza de delincuentes.

\section{d) Re-utilización de los estereotipos en los medios de comunicación}

Cualquier dictadura persigue controlar los medios de comunicación y la literatura. El régimen franquista no fue una excepción. Hasta 1966, toda la información publicada en la prensa tuvo que ser controlada y autorizada por la censura dirigida por el Ministerio de Información. Ocurrió lo mismo en relación a los libros, ya fueran estudios históricos, monografías, ensayos o novelas. Ninguna publicación pudo escapar de este control previo, con la notable excepción de las publicaciones católicas, las cuales eran sometidas a su propio control. Cualquier publicación puede considerarse como una expresión de la ideología oficial o, al menos, que no está en desacuerdo con la misma. Subrayamos la connivencia entre el régimen, los medios de comunicación y los intelectuales sobre los Gitanos.

En las monografías especializadas de los años 40 y 50, hallamos la explicación de la distinción entre los buenos Gitanos y los malos Gitanos. Los primeros son bellos, sobre todo las mujeres. Bailan flamenco, tocan la guitarra, tienen amor por su familia, la naturaleza y la libertad, y son muy religiosos. Fueron muy útiles para el turismo. Los segundos son feos, sucios. Roban, matan, luchan entre sí y se dan a la bebida. José Carlos de Luna o Manfredi Cano, quienes fueron reconocidos como especialistas sobre Andalucía y los Gitanos durante el periodo franquista, explican esta diferencia debido a su historia. Los "Buenos Gitanos” serían los verdaderos gitanos españoles que obtuvieron 
su redención gracias a España. Son aquellos que se habrían establecido en España desde muy temprano (en la antigüedad según De Luna) y habrían llegado desde el norte de África (de Luna, 1951). Los otros gitanos habrían llegado procedentes de Francia y Europa desde el siglo XV. En realidad, los primeros son la imagen ideal de los Gitanos andaluces, la misma que es utilizada por las autoridades para promover la región. Los segundos son la imagen negativa de los demás, aquellos que vivían en los barrios populares o pobres o en los suburbios cerca de la ciudad.

Generalmente, en la literatura y los artículos de prensa sobre los Gitanos hasta mediados de los años 60 y a menudo después, los viejos estereotipos como el robo de niños, la delincuencia, la suciedad se mezclaban con los nuevos prejuicios acerca del conservadurismo, las drogas y la marginalidad. Y cuando las páginas culturales de los periódicos honraban a Carmen Amaya y otras estrellas del flamenco, simultáneamente las páginas de noticias de sociedad o "sangrientas" del mismo periódico mostraban la lista de los supuestos crímenes gitanos. Lo peor hostilidad contra los Gitanos aparecía sin duda en el tabloide "El caso", el cual estaba dedicado por completo a las noticias sangrientas. De 1952 a 1981 esta publicación fue una tribuna para el odio contra los Gitanos (Marie Franco, 2000). La literatura infantil no estaba aparte. Dos ejemplos ilustran lo que podemos denominar el aprendizaje de la desconfianza hacia esta comunidad: la novela "Coliche entre los Gitanos", publicada en 1952 (García Bellver, 1952) y el cómic "Los churumbeles", que fue publicado por primera vez en 1962 en la revista DDT. La novela cuenta la historia de un joven gitano limpio y honesto, Coliche, quien vive con una mala familia de ladrones que quieren que robe, pero él no puede. Al final de la historia aprendemos que Coliche no es gitano en realidad y fue adoptado por esta mala familia. La demostración se lleva a cabo. La educación no es la cuestión, la deshonestidad está en la sangre. Es la misma idea que aparece en la saga de la familia churumbel cuya única actividad consiste en robar de todo. El humor reclamado por el autor no puede ocultar los estereotipos aplicados y especialmente el más fuerte de todos: todos los Gitanos son ladrones.

\section{e) Evolución de la posición de la Iglesia: de la hostilidad al paternalismo}

La Iglesia católica y el régimen franquista se apoyaron recíprocamente. Tan pronto como estalló la Guerra Civil, la Iglesia y todo su aparato dieron su apoyo a la insurrección nacionalista. Hasta mediados de los años 60 cada facción requería del apoyo de la otra antes de una lenta y cuidadosa separación. La actitud de la Iglesia católica hacia los Gitanos fue más compleja y gradual. Desde el siglo XVI hasta principios de 1950, la demonización y la imputación de herejía era la actitud general de los eclesiásticos. Durante el siglo XVII los ataques más duros fueron redactados por clérigos y dirigidos a los reyes españoles (Sánchez Ortega, 1991). El más famoso es el Discurso contra los 
Gitanos de Sancho de Moncada (1619). En este texto encontramos todos los estereotipos posteriores en relación al robo, el robo de niños, la prostitución, el espionaje, la pereza, las supersticiones... Este documento puede ser considerado como el acto fundador del "antigitanismo" en España. Hasta el siglo XX muchos argumentos desarrollados por Sancho de Moncada fueron reutilizados en varios libros. La Gran Redada contra los Gitanos en 1749 fue una ilustración de la alianza entre la Iglesia y el Estado en contra de esta comunidad (Gómez Alfaro, 1994). Esta incursión fue organizada por Fernando VI tras una propuesta del obispo de Oviedo Vázquez Tablada, miembro del Consejo de Castilla. Los clérigos modernos tienen una gran responsabilidad en la criminalización de los Gitanos. En el comienzo del siglo XX, el Padre Jerónimo Montes, quien fue criminólogo también representante de la escuela clásica de criminología, reprodujo la "tradición" clerical de hostilidad en sus estudios sobre la criminalidad, pero bajo la influencia de la concepción biológica racista de su tiempo (Montes, 1911:401-402).

Hasta mediados de los años 50, la Iglesia no realizó ninguna labor hacia los Gitanos. Las escasas menciones de esta comunidad en las publicaciones de la Iglesia se referían a la acción del Padre "Manjón", presentado como el maestro de los Gitanos. En realidad, Manjón había fundado en 1888 una escuela para los hijos de las familias de las Cuevas de Granada, que no eran Gitanos en su mayoría. El Padre Manjón tenía una mala opinión de los Gitanos, a quienes consideraba como una "raza" degenerada y sin cultivar (Obras Selectas, 1948:30-31), pero su figura fue utilizada en el siglo XX por la Iglesia para demostrar su implicación hacia los Gitanos sobre la base de un "error histórico" (Saenz Barrio, 1989). En realidad, otras voces clericales empezaron a escucharse a mediados de los años 50. Algunos sacerdotes de manera aislada estaban escandalizados por las terribles condiciones en que las familias gitanas vivían sobre todo en Granada cerca de las cuevas convertidas en espectáculos turísticos. En 1958 y 1961, dos de ellos, Fermín Castello y Sánchez Ocaña, escribieron ensayos en los que pedían una intervención más decidida de la Iglesia para ayudar a estas familias (Castillo, 1958; Sánchez Ocaña, 1963). Al mismo tiempo, desarrollaron la idea de que la superstición y la inmoralidad eran las causas de sus dificultades. De acuerdo a sus planteamientos, la mejor manera de actuar era por medio de una redención y una nueva evangelización. Pero por primera vez, emergió la idea de una obra social para los Gitanos. Debemos esperar hasta mediados de los años 60, para observar el comienzo de una implicación real de la Iglesia. Influenciada por el Concilio Vaticano II y el discurso a los gitanos en Pomezia (Italia) de Pablo VI 
en $1966^{5}$, la parte más liberal de la Iglesia, especialmente en Cáritas, desarrolló acciones sociales, religiosas y políticas. El paternalismo era muy fuerte y al principio pocos Gitanos participaron en las decisiones. Sin embargo, la constitución del movimiento pro gitano y la implicación de cada vez más gitanos fue la señal de la necesidad de emancipación. Juan de Dios Ramírez Heredia fue un ejemplo perfecto de la evolución de este movimiento proveniente de la Iglesia, siendo retomado por los propios Gitanos.

En el contexto de finales de los años 60, los Gitanos se convirtieron en una causa social y política, y en el signo premonitorio de una mayor demanda por la justicia social. Las diferentes aproximaciones hacia los Gitanos por parte del Estado, la Iglesia y los medios de comunicación son una ilustración de las tensiones en la vieja sociedad franquista.

El caso de la aprehensión respecto a los Gitanos del régimen franquista parece demostrar que el nacionalismo de extrema derecha no puede prescindir del racismo. En España, el mito de la hispanidad fue un intento de conciliación entre el universalismo católico y la "supremacía blanca y europea”. El carácter católico no impidió la categorización biológica racista pero la influencia de la Iglesia previno una legislación racista explícita. Más allá de la representación folclórica, las comunidades gitanas eran contempladas como un contraejemplo perfecto e integral sobre la base de la alianza entre el régimen, la prensa y la élite intelectual (gráfico 1). El caso de los Gitanos es también un ejemplo de la lenta separación de la Iglesia respecto al régimen franquista (gráfico 2).

5. «Vous êtes venus avant tout dans un monde qui ne vous méprise pas, qui ne vous persécute pas, qui ne vous exclut pas de son sein. Vous devez reconnaitre que le monde qui vous entoure nest plus celui d'il y a quelques décennies, ce monde qui vous proscrivait et vous fais ait tant souffrir. Sans éprouver de haine pour ceux qui vous ont cruellement persécutés, qui ont mené à la mort tant de vos semblables. Nous aurons une pensé espéciale pour les Tsiganes victimes des persécutions raciales. Nous prierons pour vos morts, nous demanderons à Dieu la paix éternelle pour eux et la paix terrestre pour tous les hommes de ce monde. Oui, soyez bons et justes, et reconnaissez qu'aujourd'hui la société est meilleure. Si vous préférez rester en marge de cette société, quoi qu'il vous en coûte, celle-ci cependant offre à tous sa liberté, ses lois et ses services.» «Mais ce qui aujourd'hui compte le plus, c'est une découverte différente. Vous découvrez que vous nêtes pas en dehors mais au dedans d'une autre société, visible mais spirituelle, humaine mais religieuse. Cette société vous le savez c'est l'Eglise. Aujourd'hui, mieux que jamais peut-être, vous découvrez l'Eglise.» "Loin d'être en marge vous êtes dans certains aspects, au centre, au cour de l'Eglise. Vous êtes au cour de l'Eglise parce que vous êtes pauvres et que vou savez besoin d'aide, d'instruction. L'Eglise aime les pauvres, les malheureux, les petits, les déshérités, les abandonnés.»

«Ici, dans l'Église, vous vous apercevrez que vous êtes non seulement des compagnons, des amis, mais des frères. Et ce lien de fraternité n'existe pas seulement entre vous et Nous, qui vous accueillons aujourd'hui, mais sur le plan chrétien, il existe avec tous les hommes.»

"Nous voudrions que cette rencontré exceptionnelle ait pour résultat de vous faire penser à la Sainte Église à la quelle vous appartenez; de mieux la connaître, de mieux l'apprécier, de mieux l'aimer. Nous voudrions que cette rencontré éveille en vous la conscience de ce que vous êtes. Chacun de vous doit se dire: je suis chrétien, je suis catholique. Et si te lou tel dentre vous ne peut pas dire cela parce qu'il nia pas cette chance, qu'il sache que l'Église catholique l'aime, le respecte et l'attend lui aussi. Qu'il veuille bien regarder l'Eglise avec sincérité et bonté.» 
Gráfico 1: Los Gitanos, un contraejemplo social absoluto en la España franquista

\begin{tabular}{|c|c|}
\hline Estándar social franquista & $\begin{array}{l}\text { Representación de los Gitanos por las autoridades (A), medios de } \\
\text { comunicación (M) y la Iglesia (C) }\end{array}$ \\
\hline Culto al trabajo & Pereza (A.M.C) \\
\hline Respeto por la propiedad privada & Robo (A.M.C) \\
\hline Fe católica & $\begin{array}{l}\text { Superstición y prácticas de adivinación (A.M.C. hasta Pomezia en } \\
1965 \text { para la Iglesia) }\end{array}$ \\
\hline Respeto por las leyes & Rechazo de las normas (A. M.) \\
\hline Solidaridad nacional & Solidaridad racial (A.M.C.) \\
\hline Patriotismo y nacionalismo & Personas sin Estado \\
\hline Honestidad & Deshonestidad (A.M.C) (hasta Pomezia en 1965 para la Iglesia) \\
\hline Limpieza & Suciedad (A.M.C.) \\
\hline Moralidad & Engaño (A.M.C.) (hasta Pomezia en 1965 para la Iglesia) \\
\hline Castidad & Promiscuidad sexual (A.C.) \\
\hline Raza hispana espiritual & Raza Gitana biológica (A. P.) \\
\hline Mujeres escrupulosas & Mujeres disolutas (A.P.) \\
\hline Niños sensatos & Gitanillos retrasados (A.P.C.) \\
\hline
\end{tabular}


Gráfico 2: Evolución y uso público de la representación de los Gitanos por las autoridades, los medios de comunicación y la Iglesia

\begin{tabular}{|c|c|c|c|}
\hline & $\begin{array}{l}\text { Autoridades } \\
\text { públicas }\end{array}$ & $\begin{array}{c}\text { Medios de } \\
\text { comunicación }\end{array}$ & Iglesia \\
\hline 1940 & & & \\
\hline & Raza de delincuentes & & $\begin{array}{l}\text { Gitanos supersticiosos } \\
\text { y/o heréticos }\end{array}$ \\
\hline 1950 & (Artista Flamenco) & $\begin{array}{l}\text { Artista Flamenco } \\
\text { Gitanos supersticiosos }\end{array}$ & \\
\hline 1960 & & & Lostbrother \\
\hline 1965: Pomezia & $\begin{array}{l}\text { Delincuentes Sociales } \\
\text { Marginal }\end{array}$ & $\begin{array}{l}\text { Delincuentes Sociales } \\
\text { Artista Flamenco } \\
\text { Marginal }\end{array}$ & Buen Salvaje y/o Marginal \\
\hline
\end{tabular}




\section{REFERENCIAS BIBLIOGRÁFICAS}

Alvarez Chillida, Gonzalo (2002) El antisemitismo en España. La imagen del judío (18122002). Madrid: Ed Marcial Pons.

Andres-Gallego, José y Pazos, Antón (1998) “Histoire religieuse de l'Espagne”. Cerf, coll. Histoire religieuse de l'Europecontemporaine. Paris.

Asséo,Henriette (1994) Les Tsiganes une destinée européenne. Gallimard, Coll. Découverte. Fernández Fernández, M.D.; Flor Heredia, M. y Rodríguez Padilla, E. (2009) El pueblo gitano en la guerra civil y la posguerra. Andalucia oriental. Granada: Asociación de Mujeres Gitanas-Romi.

Barrachina, Marie-Aline (1998) Propagande et culture dans l'Espagne franquiste, 19361945. ELLUG, Université Stendhal de Grenoble.

Bennassar, Bartolomé (1992) Histoire des Espagnols, VI ${ }^{e}-X X^{e}$ siècle. Robert Laffont, Coll. Bouquins.

Castillo, Fermín (1958) Los Gitanos. Zaragoza: La editorial.

Comandante Casillas (1965) “Gitanos, caballerías y sus transmisiones”, Guardia Civil, n 253, pp. 50-52.

Dirección Nacional de Apostolado Gitano (1968) Informe sobre los Gitanos, inédito.

de Luna, José Carlos (1951) Gitanos de la Bética. Madrid: Epesa.

Dr. Echalecu y Canino (1945) "Los institutos de Biología criminal”, REEP, n7, pp. 2-8.

Franco, Marie (1995) El Caso, 1952-1962 :récits fantasmatiques et écriture littéraire dans le fait divers espagnol. Thèse de doctorat, Université de la Sorbonne nouvelle, Paris III. Franco, Marie (2000) «Le Gitan honni». En Jean René Aymes y Serge Salaun (coords.) Etre espagnol. Paris: Presse de la Sorbonne nouvelle, pp. 337-339.

Franco, Marie (2005) Le sang et la vertu - faits divers et franquisme (ElCaso 1952-1962). Madrid: La Casa de Velázquez.

Garcia Bellver, Carmen (1957) Coliche entre los Gitanos. Valencia: Aitana.

Gay y Blasco, Paloma (1999) Gypsies in Madrid. Sex, Gender and the Performance of Identity. Oxford-New York, Berg.

Gómez Alfaro, Antonio (1994) La grande rafle des Gitans. Paris: CRT/CRPD Midi Pyrénées Collection Interface.

Guerra, Valentín (1944) "El vagabundo desde el punto de vista policial", Guardia Civil, $\mathrm{n}^{\circ} 5$, pp. 51-54.

Hoyos de Sainz, Luis (1951) "La raza, las razas y los subgrupos en España", Antropología y Etnología, nº y 5, pp. 321-346.

Jiménez Rubio (1948) "Servicio rural, estados próximos y peligrosos al delito", Guardia Civil, $n^{\circ} 46$, pp. 18-21.

Leblon, Bernard (1964) “Les Gitans dans la péninsule ibérique, I”. Etudes Tsiganes, marsjuin 1964. 
Leblon, Bernard (1978) «Recherches historiques en Espagne». Etudes Tsiganes, nº 2, $24^{\mathrm{e}}$ année, juin.

Leblon, Bernard (1982) Les Gitans dans la littérature espagnole. Toulouse: Institut d'Etudes Hispaniques et Hispano-Américains, Université de Toulouse-Le Mirail.

Leblon, Bernard (1985) Les Gitans d'Espagne. Paris: Presse Universitaire de France.

Leblon, Bernard (1990) Musiques tsiganes et flamenco. Paris: L'Harmattan, col. Tsiganes. Martin, David (2005) «Gitanos en la Guerra civil espanola». I Tchatchipen, nº51, pp. 2736.

Moncada, Sancho de (1619) "Discurso contra los Gitanos" en Restauración política de España y deseos públicos. Madrid: Luis Sánchez.

Montes, Jerónimo (1911) Precursores de la ciencia Penal en España, Estudios sobre el delincuente y las causas y remedios del delito. Madrid: Ed. Librería General de Victoriano Suarez.

Obras selectas de D. Andrés Manjón (1948) Patronato de las Escuelas del Ave María, Imprenta talleres Penitenciarios de Alcalá de Henares.

Payne, Stanley (1992) Franco. El perfil de la historia. Madrid: Espasa-Calpe.

Rothea, Xavier (2007) "Kriminal biologie ethygiénisme raciale, l'influence nazie dans l'appréhension des Gitans dans l'Espagne franquiste". Etudes Tsiganes, $\mathrm{n}^{\circ} 31$.

Sabater Tomás, Antonio (1962) Gamberros, homosexuales, vagos y maleantes. Barcelona: Hispano-América, Sabater Tomás, Antonio (1972) Peligrosidad social y delincuencia. Barcelona: Ediciones Nauta.

Saenz Barrio, Oscar (1989) “Un error histórico: Manjón educador de Gitanos”. Cuadernos de pensamiento, $\mathrm{n}^{\circ} 3$, pp.55-70.

Sánchez Ocaña, Juan (1963) Granada y sus Gitanos. Granada: Ave María.

Sánchez Ortega, Helena (1978) “Les Gitans espagnols face à l'inquisition”. Etudes Tsiganes, $\mathrm{n}^{\circ} 2,24^{\mathrm{e}}$ année.

Sánchez Ortega, Helena (1984) "Hechizos y conjuros entre los Gitanos y los no-Gitanos". Cuadernos de historia moderna y contemporánea, n5, pp. 83-136.

Sánchez Ortega, Helena (1988) La inquisición y los Gitanos. Madrid: Taurus Ediciones. Sánchez Ortega, Helena (1991) "La oleada anti-gitana del siglo XVII". Espacio, tiempo y forma, Serie IV, Historia moderna, t. IV, pp. 71-12.

San Román, Teresa (1976) Vecinos Gitanos. Madrid: Akal.

Serrano Garcia, Pedro (1945) "Vocabulario del caló delincuente", Policía, n³7.

Thede, Nancy (1999) Gitans et flamenco, les rythmes de l'identité. Paris: L'Harmattan. 